\title{
How much complexity do we need in coupled benthic ecosystem and early diagenesis model?
}

\author{
STANLEY I NMOR ${ }^{1}$ AND KARLINE SOETAERT ${ }^{2}$
}

${ }^{1}$ LSCE/CNRS CEA UVSQ University Paris-Saclay

${ }^{2}$ Royal Netherlands Institute of Sea Research (NIOZ)

Presenting Author: stanley.nmor@1sce.ipsl.fr

In shallow marine ecosystems, the microphytobenthos is considered as an important driver in modulating several biogeochemical processes through their activities. Models that simulate the cycling of nutrients through primary producers and subsequent mineralization of organic matter are important tools for understanding how ecosystem function. However, their inclusion in benthic ecosystem models is only discussed in a few studies. In this investigation, we constructed a new numerical model that encompasses the "state of the art" understanding of important benthic processes to explore the role of microphytobenthos (MPB) and physical forcing on sediment biogeochemistry. Revising previous model formulations that entail early diagenesis processes and micro-algae physiology, our effort introduced mechanistic formulations that detail these complex dynamics without sacrificing simplicity and realism. To that end, we apply this new model on a variety of ecological scenarios to highlight how the benthic ecosystem and its underlying biogeochemical processes is being modulated by the different biological and physical factors. Our results show that inclusion of MPB can introduced fine-scale diurnal variability in several processes. Total mineralization was enhanced by $19.8 \%$ in the presence of MPB because of the input of fresh labile organic matter. The depth of the oxic layer was increased, resulting in an increased coupled nitrification-denitrification process. Imposing the influence of tide effect resulted in high surface nutrient (ammonium, nitrate, phosphate) accumulation when the sediment was exposed, and that was quickly exchanged with the overlying water upon immersion. Thus, the sediment acts as a source of nutrient to the water column. The different scenarios explored in the model showed the complex behaviour exhibited by MPB under dynamically changing environments which have significant biogeochemical ramifications. 